Claremont Colleges

Scholarship@ Claremont

Pomona Faculty Publications and Research

Pomona Faculty Scholarship

$1-1-1993$

\title{
Nanoscale study of the as-grown hydrogenated amorphous silicon surface
}

David M. Tanenbaum

Pomona College

G. C. Stutzin

R.M. Ostrom

Alan C. Gallagher

\section{Recommended Citation}

The following article appeared in "Nanoscale study of the as-grown hydrogenated amorphous silicon surface," G. C. Stutzin, R. M. Ostrom, Alan Gallagher, and D. M. Tanenbaum, J. Appl. Phys. 74, 91 (1993), DOI:10.1063/1.355203 and may be found at http://link.aip.org/link/?JAPIAU/74/91/1 


\author{
G. C. Stutzin, ${ }^{\text {a) }}$ R. M. Ostrom, and Alan Gallagher ${ }^{\text {b) }}$ \\ Joint Institute for Laboratory Astrophysics, University of Colorado and National Institute of Standards \\ and Technology, Boulder, Colorado 80309-0440 \\ D. M. Tanenbaum \\ National Renewable Energy Laboratory, Golden, Colorado 80401
}

(Received 7 December 1992; accepted for publication 22 March 1993)

\begin{abstract}
A scanning tunneling microscope has been used to study the topography of the as-grown surface of device-quality, intrinsic, hydrogenated amorphous silicon deposited by $\mathrm{rf}$ discharge from silane. The substrates were atomically flat, oxide-free, single-crystal silicon or gallium arsenide. No evidence for island formation or nanoscale irregularities was seen in studies of 100 -A-thick films on either silicon or gallium arsenide. The topography of 1000 - and 4000 - $\AA$-thick films has much variation; many regions can be characterized as "rolling hills," but atomically flat areas have also been observed nearby. Generally, it appears that surface diffusion plays a role in smoothing the film topography. In most regions, the observed slopes were $10 \%$ or less from horizontal, but some steep-sided valleys, indicating incipient voids, were observed. The effect of the finite size of the scanning tunneling microscope probe tip is considered; this has an effect on the observed images in some cases.
\end{abstract}

\section{INTRODUCTION}

Thin films of hydrogenated amorphous silicon ( $a$ $\mathrm{Si}: \mathrm{H})$ are used in photovoltaic cells and a variety of microelectronics applications, notably, imaging sensors and flat panel displays. Although $a$-Si:H may be deposited by reactive sputtering, thermal chemical vapor deposition, and other methods, it is mostly commonly deposited by rf plasma-enhanced chemical vapor deposition (PECVD), the method employed here. The semiconducting properties, and particularly the light-induced degradation, ${ }^{1}$ of these films are strongly dependent on the discharge characteristics. This has prompted many measurements and models of the discharges and the associated gas phase or plasma chemistry. Some of the plasma-chemistry models include both surface reactions that lead to film growth, as well as possible causes for variation in film porosity. ${ }^{2-7}$ However, due to an absence of detailed surface data these models are highly simplified. The models do not explain the varying properties of relatively dense, device-quality films, which are of primary importance. We believe that a detailed understanding of the mechanism of film growth, including sources of film inhomogeneities, is now necessary. In essence, the last 15 years have been filled with many studies of the gas phase and of the grown films, but very few regarding the growing surface. The present study is directed toward filling this gap by studying the atomicscale topography of the growing surface.

A variety of methods have led to a recognition that $a-\mathrm{Si}: \mathrm{H}$ films are inhomogeneous. Knights and Lujan showed, using the scanning electron microscope (SEM) and the transmission electron microscope (TEM), that films deposited under "physical deposition" conditions

\footnotetext{
a) Present address: Siliconix, Inc., 2201 Laurelwood Ave., Santa Clara, CA 95056.

b) Staff Member, Quantum Physics Division, National Institute of Standards and Technology, Boulder, CO 80309-0440.
}

consisted of microcolumns and had poor electronic quality. ${ }^{8}$ However, they did not detect, with $\sim 10 \AA$ resolution, observable microstructure in good electronic quality films deposited under the "chemical deposition" conditions (low-power if discharge in pure silanc) now in essentially universal use. In contrast, Ross et al. did observe microstructures and columnar growth in high-quality films. ${ }^{9}$ In addition, NMR measurements have now shown that a great deal of clustered hydrogen exists in these high-quality films, suggesting regions of low Si density or "voids." 10 (The average Si density in $a-\mathrm{Si}: \mathrm{H}$ is $\sim 95 \%$ of that in crystal Si.) Small-angle $\mathrm{x}$-ray scattering (SAXS) has now also reported the presence of these voids. ${ }^{11}$ There is every reason to believe that these voids are extremely important to the electronic properties and stability of $a-\mathrm{Si}: \mathrm{H}$ films. A major thrust of this study is to look for these voids and determine their effects on the growing film surface.

Several methods have been employed to study characteristics of the $a$-Si:H film surface, including the scanning tunneling microscope (STM) method employed here. Ellipsometry is perhaps the oldest and most widely utilized method. ${ }^{12,13}$ This detects spatial irregularities in surface growth rate and the existence of surface layers with varying indexes of refraction. It has excellent thickness resolution $(\sim 10 \AA)$, and detects an average property over a large surface area. Similarly, infrared reflection absorption spectroscopy of $\mathrm{SiH}_{x}$ species during growth has been utilized. ${ }^{14}$ At this laboratory, the thickness of the H-rich surface layer of various $a$-Si:H films was established by measuring hydrogen evolution as we slowly sputtered away the surface. ${ }^{15}$ Both of these measurements yield spatial averages of surface properties.

Several groups have employed the scanning tunneling microscope (STM) to study the surface of the film. Kazmerski reported atomic resolution on cleaved surfaces and was able to distinguish the silicon atoms from the hydrogen atoms by using STM current voltage spectros- 

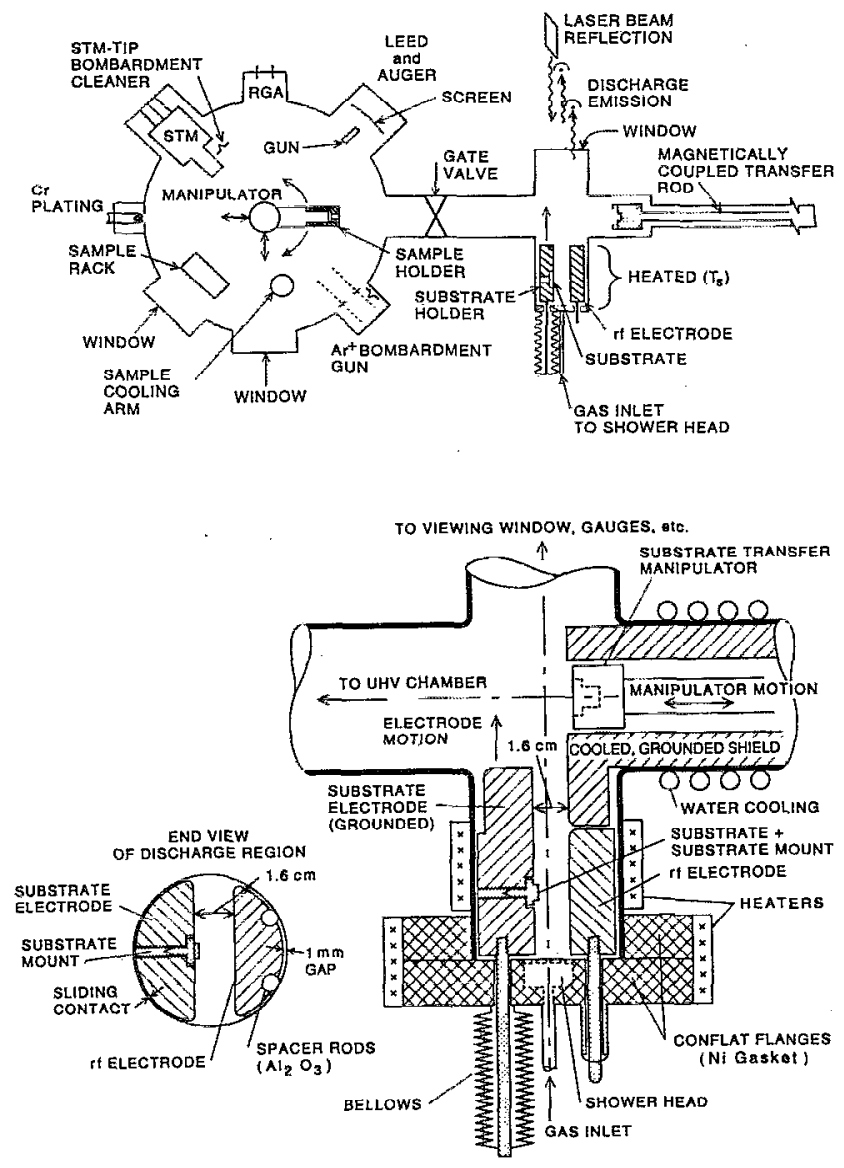

FIG. 1. (a) Schematic diagram of the overall apparatus. (b) Diagram, to scale, of the rf-discharge film-deposition chamber.

copy with photon biasing. ${ }^{16}$ Wiesendanger et al. studied the surface of 5000- $\AA$-thick films after "gentle $\mathrm{Ar}^{+}$bombardment cleaning" and found the surface to be almost atomically flat. ${ }^{17}$ They also observed, in contrast to Kazmerski, an essentially constant surface work function. (Small, localized variations of the work function were measured but the cause was not clear.) In both studies, highly doped films were studied to enhance conductance of the tunneling current. Jahanmir et al. performed surface modifications of $a-\mathrm{Si}: \mathrm{H}$, in air, using a STM.$^{18}$ Hartmann et al. have "written" electrically active lines in highly doped $a$-Si:H on top of single-crystal silicon. ${ }^{19}$ Vandentop et al. used an STM to study the initial stages of growth of amorphous hydrogenated carbon ( $a-\mathrm{C}: \mathrm{H})$ on highly oriented pyrolytic graphite and single-crystal silicon substrates. ${ }^{20}$

In this paper we present results from an STM study of the surface topography of as-grown, intrinsic $a-\mathrm{Si}: \mathrm{H}$ deposited by PECVD from pure silane on atomically flat, oxidefree crystalline $\mathrm{Si}$ and $\mathrm{GaAs}$ substrates under devicequality deposition conditions. The film thickness ranges from 100 to $4000 \AA$.

\section{EXPERIMENT}

A schematic diagram of the STM chamber and the combination load-lock and deposition chamber is shown in
Fig. 1(a). Details of the deposition region are shown in Fig. 1(b). The two chambers were connected by a gate valve so that as-deposited films could be observed in the STM without exposure to air. The (grounded) substrate electrode in Fig. 1(b) could be moved under vacuum for sample exchange, while the rf electrode was stationary. Small gaps between the if electrode and grounded walls largely confined the discharge to the $5-\mathrm{cm}$-square, $1.6-\mathrm{cm}$ deep region between the electrodes. The discharge was electrically asymmetric. The arm of the cross containing the deposition apparatus was usually heated to $\sim 300^{\circ} \mathrm{C}$ by band heaters on that arm, while the four adjacent arms of the cross were water cooled. Due to these nearby cooled surfaces, the substrate temperature $T_{s}$ was $90 \%$ of the hotwall temperature; $T_{5} \sim 250^{\circ} \mathrm{C}$ for all data presented here. The gas fiow in the deposition system was parallel to the electrode surfaces and originated with a "showerhead." The deposition chamber also contained a magnetically coupled transfer arm, to carry samples between chambers, a capacitance manometer, ion gauge, and windows. The turbomolecular-pumped chamber had a base pressure below $10^{-5} \mathrm{~Pa}$ (1 Torr $=133.32 \mathrm{~Pa}$ at deposition temperature. The UHV chamber was ion pumped with a base pressure of $\sim 3 \times 10^{-8} \mathrm{~Pa}$, mostly $\mathrm{H}_{2}$. We used an STM of the Lyding configuration, ${ }^{21}$ using chemically etched tungsten tips that were cleaned by electron-bombardment heating.

To ensure that observed film-surface topography is due to the film only, and as free of impurities as possible, the following procedure was used. A (100) or (111), n- or $p$-type, $\sim 0.01 \Omega \mathrm{cm}$, silicon wafer was wet cleaned with a standard Shiraki etch, ${ }^{22}$ mounted in the silicon sample holder, and loaded into the load-lock chamber. When the pressure reached $\sim 10^{-5} \mathrm{~Pa}$ in that chamber, the sample holder was transferred to the UHV chamber and degassing was begun by passing current through the crystal. Usually, the sample was degassed for at least $12 \mathrm{~h}$ at $\sim 500^{\circ} \mathrm{C}$. The sample was then flashed to $850^{\circ} \mathrm{C}$ for $5 \mathrm{~min}$ to remove the oxide, with the pressure below $2 \times 10^{-7} \mathrm{~Pa}$ at all times. A low-energy electron diffraction (LEED) pattern was taken to ensure that the sample was well cleaned and reconstructed, and after $\sim 90 \mathrm{~min}$ of cooling the sample was loaded into the STM. STM images were taken in several regions separated by macroscopic distances to check that the sample was indeed atomically flat. This was generally the case, but some 4000 - $\AA$-thick films were deposited on $\mathrm{Si}$ crystals with $\sim 20 \AA$ irregularities, which was deemed unimportant for such film thickness. The tunneling parameters on the crystalline surface were usually $-3 \mathrm{~V}$ and 200 pA. Tunneling voltages will refer to the sample voltage relative to the probe voltage (virtual ground). Although the STM generally did not resolve atomic sites, height res-

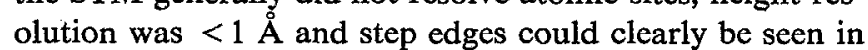
these images. The sample was then transferred to the substrate electrode in the deposition chamber, which by this time had been degassed at temperatures $\sim 50^{\circ} \mathrm{C}$ higher than the deposition temperature. The sample was held in the grounded electrode for at least $10 \mathrm{~min}$ before deposition, to ensure that the substrate temperature reached $\sim 250^{\circ} \mathrm{C}$. For gallium arsenide substrates, the procedure 
was much simpler. A $\sim 0.4$-mm-thick, 3-mm-wide $p$-type, $\sim 0.001 \Omega \mathrm{cm}$ wafer initially protruded $\sim 7 \mathrm{~mm}$ from a sample holder, and was cleaved in the deposition chamber to expose the (110) face. Thereafter the sample was not heated or specially cleaned in any way. The exposed surface protruded $\sim 1 \mathrm{~mm}$ above the holder, and contained large, atomically flat areas on the cleaved face. The $\mathrm{Si}$ and GaAs sample surfaces protrude above their holders 1-2 $\mathrm{mm}$, which is deemed insignificant compared to the $1.6-\mathrm{cm}$ electrode gap.

Once the substrate was mounted in the deposition system, silane flow was initiated for $\sim 20$ min prior to discharge initiation. A gate valve between the deposition chamber and the turbomolecular pump was set manually to regulate the pressure. The silane flow and pressure were $11 \mathrm{sccm}$ and $72 \mathrm{~Pa}(540 \mathrm{mTorr})$ at $250^{\circ} \mathrm{C}$; gas dwell time in the discharge was $0.25 \mathrm{~s}$.

A single spark from a Telsa coil to a separate, isolated feedthrough initiated the discharge once the rf voltage had been turned on to $\sim 130 \mathrm{~V}_{p p}$. Due to the difficulty of measuring if power delivered to the gas, deposition rate rather than if power was measured. This was made particularly easy by calibration measurements in this chamber, which determined that the discharge optical emission detected with a $\mathrm{Si}$ photodiode was very well correlated to the film deposition rate $R$ over a wide range of discharge powers and substrate temperatures. Thus, the photodiode signal was used to determine $R$ and also to check for stability during the film growth. For the films reported here $R \cong 1.7$ $\AA / \mathrm{s}$, and $\sim 5 \%$ of the silane was depleted by the discharge. After the desired amount of film had been deposited, the rf generator was switched off, the turbomolecular-pump gate valve was opened, the flow of silane was shut off, and the sample was transferred from the grounded electrode to the transfer arm. The pressure in the deposition chamber reached the low $10^{-5} \mathrm{~Pa}$ range in $\sim 5 \mathrm{~min}$, at which time the sample was transferred to the UHV chamber and further cooled by contacting a cold finger for $\sim 30 \mathrm{~min}$. The sample was moved to the STM and imaging commenced, although thermal STM drift was often noticeable for the first few hours. We observed no effects correlated with vacuum exposure time. This behavior reaffirms other data which indicate that the as-grown surface of $a-\mathrm{Si}: \mathrm{H}$ is extremely inert. ${ }^{5,23}$

\section{TUNNELING CONDITIONS}

An STM is normally operated with $10 \mathrm{pA}$ to $1 \mathrm{nA}$ tunneling current, passing through a 3-8 $\AA$ vacuum gap between the end of the probe and the sample. For atomic scale resolution this current passes through a region on the order of $5 \AA$ diameter. This enormous electron current density $\left(\sim 10^{5} \mathrm{~A} / \mathrm{cm}^{2}\right)$ is easily sustained by metal samples without significant surface damage or voltage drop. However, in the case of crystalline semiconductors, it generally requires $>2 \mathrm{~V}$ applied between sample and metal probe to overcome the band bending and spreading rcsistance in the semiconductor. In the case of $a$-Si:H films, which are much less conductive than crystal semiconductors even when doped, it is not apparent how much voltage drop will occur in the film during tunneling. Consequently, we have studied the dependence of constant current tunneling and apparent surface topography on applied voltage and feedback current set point. Too low a voltage or too high a current results in surface damage and material transfer between sample and probe by direct contact of the probe and sample, whereas too high a voltage induces sample and probe surface changes by atomic migration. We typically find that $\sim 5 \mathrm{~V}$ and $20 \mathrm{pA}$ is the best compromise for a 4000 - $\AA$-thick film ( $\sim 3 \mathrm{~V}$ for a $100-\AA$-thick film), where $20 \mathrm{pA}$ is the lowest current that gives adequate bandwidth and signal/noise for our STM. We believe that a significant fraction of this voltage typically appears across the tunneling gap, and the remainder induces the necessary current density in the $a-\mathrm{Si}: \mathrm{H}$. The low-field conductivity of intrinsic $a$-Si:H is totally insufficient to sustain the current density required for STM imaging, and a very large electric field induces the necessary current flow in the film. (Intrinsic $a$-Si:H has a low-field resistivity $\rho>10^{9}$ $\Omega \mathrm{cm}$.) We have also investigated light biasing (up to $\sim 0.1 \mathrm{~W} / \mathrm{cm}^{2}$ ) to increase the film conductivity, but this generally has a rather small effect due to the large carrier density already present in the sample.

Due to the large scale of film surface roughness compared to tunneling gaps, we always scanned the surface in the constant-current tunneling mode. However, we frequently interspersed this with current, $i$, versus probeheight, $z$, data at a point with constant voltage $V$. If the probe penetrates the sample, as occurs with insufficient voltage or excessive current, this produces a very gradual and often irregular decrease in $i$ with increasing $z$. If excessive voltage is applied at constant current, this induces irregular $z$ fluctuations versus time at a point due to atomic and bonding rearrangements. If the tungsten probe is "dirty," or covered with nonmetallic atomic layers such as oxide or $\mathrm{Si}$, then $i(z)$ again falls relatively gradually as $z$ is increased. When the probe was clean and current and voltage conditions were optimized, we observed stable $z(t)$ at constant $i$, and $i(z) \propto \exp \left(z / z_{0}\right)$, where the scale height $z_{0}$ is $<2 \AA$. This rapid current variation with $z$ indicated the desired tunneling through a small vacuum gap between sample and probe, and that the topography obtained from a constant-current scan closely represents the actual surface shape. Different surface states at different sample positions can still produce apparent height variations, but these are expected to be small $(<2 \AA)$ under these circumstances. All data presented below were obtained under conditions that produced $z_{0}<1.5 \AA$ and "quiet" $i(t)$.

From the standpoint of STM tunneling theory, it is interesting that the sample voltage necessary to obtain equivalent vacuum tunneling [based on exponential $i(z)$ into 4000 - $\AA$-thick films] varied between -1.5 and $-6 \mathrm{~V}$ at different times (with the probe effectively grounded). This is clearly related to atomic-scale changes in the shape of the probe and its coverage by $a-\mathrm{Si}: \mathrm{H}$ debris. In a variety of instances we scanned surface regions at very different sample voltages, confirming that the detected topography was normally independent of the voltage. However, severe 


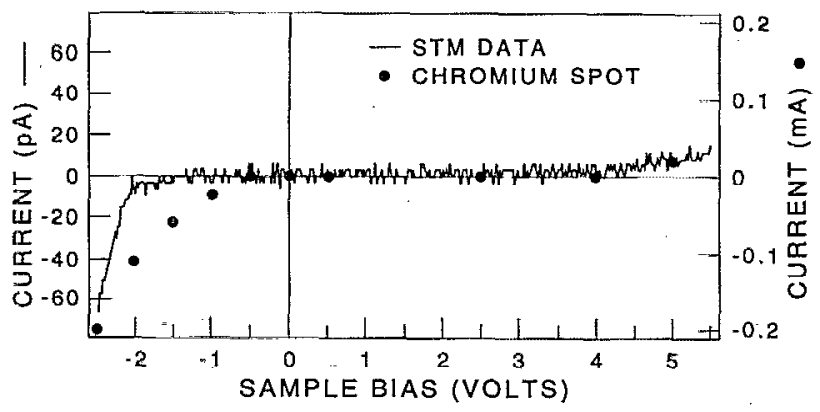

FIG. 2. Two examples of current voltage spectroscopy on $4000 \AA$ a-SiH films deposited on crystal silicon. The left axis scales the STM data, while the right side scales data for a $0.02 \mathrm{~cm}^{2}$ chromium spot evaporated on the film surface.

height fluctuations related to probe instabilities sometimes occurred outside a narrow voltage range.

Current-voltage $(I V)$ curves obtained with $z$ fixed at any point on the $4000 \AA$ film were asymmetric. As seen in Fig. 2, the positive sample voltage required to generate a given current is several volts larger than the negative voltage necessary to achieve the equivalent current. This behavior occurred for 0.02-3 nA tunneling current, and was independent of the sign of the substrate doping. Qualitatively similar n-type Shottky-diode behavior is shown in Fig. 2 from chromium dots evaporated on the exposed surface of the same $a-\mathrm{Si}: \mathrm{H}$ film, with ohmic contact to the backside of the crystalline substrate made by diffusing indium into the silicon. This simulates the operation of the STM, with the chromium dots effectively replacing the STM probe. An equivalent current density was obtained if one assigned an effective area of $\sim(800 \AA)^{2}$ to the STM current. A reasonable interpretation of the forward-bias STM current is thermionic electron emission over the low barrier in the $a-\mathrm{Si}: \mathrm{H}$, accompanied by tunneling through the high, thin vacuum barrier. The increased $a-\mathrm{Si}: \mathrm{H}$ barrier under reverse bias then limits the reverse current, yielding Shottky-diode-like STM behavior. Similar asymmetric behavior has been reported for tunneling into crystal Si (Ref. 24) and H-terminated Si (Ref. 25) with opposite dependencies on doping.

In order to further verify that the observed surface topography of this highly resistive film was a valid representation of the surface, we scanned some areas of the $4000-\AA ̊-t h i c k$ films at several sample voltages $(3-6 \mathrm{~V})$ and with varying illuminations with a $633-\mathrm{nm}$ laser beam. (The beam intensity inside the film was estimated at $\sim 10 \mathrm{~mW} /$ $\mathrm{cm}^{2}$. This intensity normally lowers the low-field resistivity from $>10^{9}$ to $<10^{5} \Omega \mathrm{cm}$.) Essentially identical topographies were obtained at the different voltages, and in most instances with different illuminations as well. However, switching on the illumination caused the STM probe to back away by as little as $1 \AA$ or as much as $20 \AA$ at different times and at different locations. We suspect this is due to varying probe and film surface states, some of whose occupancy may be light sensitive. It is important to note that this can be taken as a worst case limit of local elec-

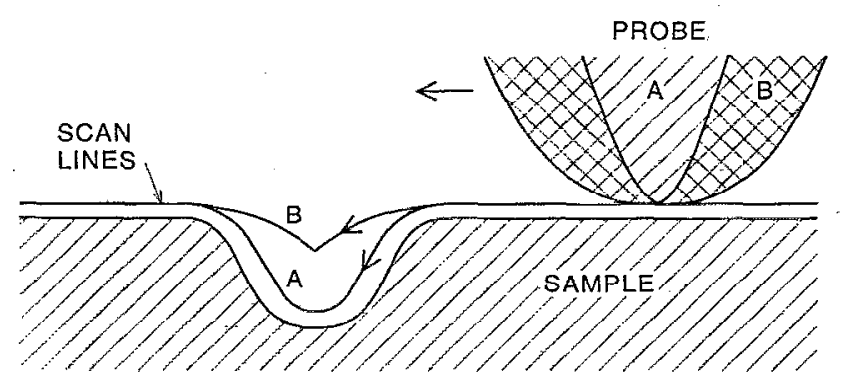

(a)

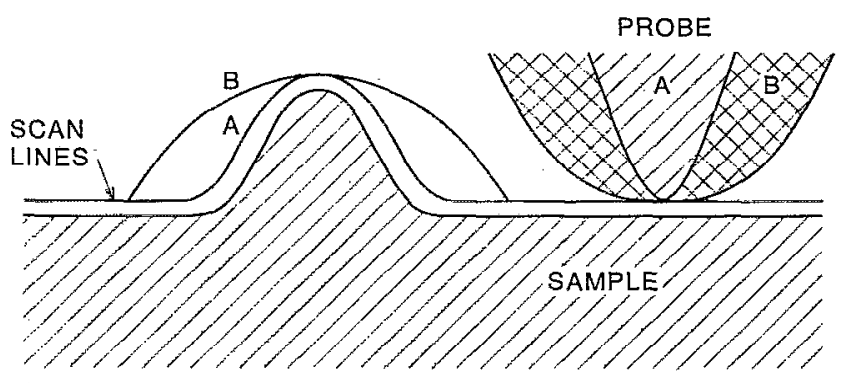

(b)

FIG. 3. Examples of the probe-sample contact transform that occurs in scanning tunneling microscopy. The lines labeled A and B are the recorded scan lines that result from scanning probe $A$ or $B$ across the sample at a fixed separation: (a) dispiays these effects in a valley while (b) represents the effects on a protrusion.

tronic effects on our topographic data, and cannot represent the much larger roughness we measure. Data reported here were obtained under circumstances where the illumination had a relatively minor effect on the tunneling gap, and did not significantly alter the observed topography.

The recorded topography represents a nonlinear contact transform of the probe surface with the sample surface, at a fixed spacing of typically $\sim 5 \AA$. This is less important in studies of essentially atomically flat crystal surfaces, as the surface height varies by only a few $\AA$. However, it is crucial when looking for $\mathrm{nm}$ size irregularities and incipient voids on the surface of $a-\mathrm{Si}: \mathrm{H}$ films. Examples of this contact transform are shown in Fig. 3, for an idealized probe shape. Note that sample protrusions appear broadened, but with true height, while sample valleys appear with decreased width and sometimes decreased depth. (As can be seen in the figure, only the ratios of probe diameter to feature dimensions are relevant. How: ever, it appears that our probe ends typically had a radius of $\sim 20 \AA$.) More detailed analysis of image distortions by probe shapes have been reported. ${ }^{26,27}$ We have considered these effects in our data analysis, and some examples will be given below. If the narrowest, steepest hills recorded by the STM were the result of a delta function type feature on the surface, these hills would be upside down (inverted) images of the probe, ignoring the 5-10 $\AA$ tunneling gap. If the tunneling gap is included, or the surface feature is broader, the probe must be even narrower. We use cross sections through the steepest, narrowest hills observed in a given set of scans to define an upper limit to the probe resolution. This cross section can then be inverted and 
considered as the outer limit to the probe plus tunnelinggap shape, as seen in Fig. 3(b). This inverted cross section (ICS) can then be compared to any features on other scans. If the ICS is much narrower than any scan feature, then the scan topography closely resembles the actual topography. If desired, one can estimate that hills will appear broadened by $\sim 50 \%$ of the ICS width, and valleys will appear narrowed by the same amount. The end of most probes, as seen in the ICS obtained here, are relatively blunt on a $\mathrm{nm}$ scale. To see how this influences images of surface valleys or pits, we have shown in Fig. 3(a) two probe sizes transformed with one valley and the resulting scan lines. Note that the scan line has a flat bottom if the probe fits in, whereas it has a cusp-shaped bottom if the probe is too wide. We use this fact to look for steep-sided, narrow holes and valleys; examples will be given below.

\section{RESULTS}

Topography data are presented for 100-, 1000-, and 4000 - $\AA$-thick films, all deposited at $250^{\circ} \mathrm{C}$ substrate temperature and $\sim 1.7 \AA / \mathrm{s}$ deposition rate. In each case data were taken from $\sim 30$ min to a week after deposition, and no indications of systematic changes were found over this time interval in the UHV chamber. However, apparent fine-scale topography changes did sometimes result from varying tunneling voltage, from probe-tip cleaning by voltage pulsing, by illuminating the film, and occasionally during repeated scans without any deliberate changes in conditions. In addition, many scans exhibited scan-direction streaks and height steps (usually 1-10 $\AA$ ), due to temporary offsets in the tunneling gap. (These are easily distinguished from actual topography by their direction and their occurrence in single scan lines.) These height steps are attributed to changes in the atomic arrangements and surface states on the probe. The $a-\mathrm{Si}: \mathrm{H}$ film is not very robust, compared to crystal Si or metals, and it appears that film components are very easily transferred to the probe during approach and scanning. We suspect that this debris is loosely bonded to the $\mathrm{W}$ probe, and is easily shifted around by the strong fields and high current densities (electrophoresis) at the tunneling point. The data presented here are selected from periods when more stable and reproducible scans were obtained. Surface topographies inferred from the noisy periods are consistent with these data, and it appears that the topographies reported here are indicative of the entire surface.

\section{A. 100-Å-thick film}

Two surface regions of a $100-\AA$-thick film, deposited onto vacuum-cleaved GaAs (110), are shown in Fig. 4. These surface regions are flat within two atomic layers with rms height variations of $\sim 2 \AA$, and are typical of those observed. Many of the (typically $\sim 3 \AA$ high and 40 $\AA$ wide) lumps in Fig. 4 did not reproduce well in repeated scans, and one can see streaks and "shelves" of $\sim 1 \AA$ height in the $x$ direction in both scans. These result from residual probe-tip instabilities, and they suggest that one should not overly interpret features $<3 \AA$ high in Fig. 4 . Some 10-20 $\AA$ high hills of $\sim 50 \AA$ width were very occa-

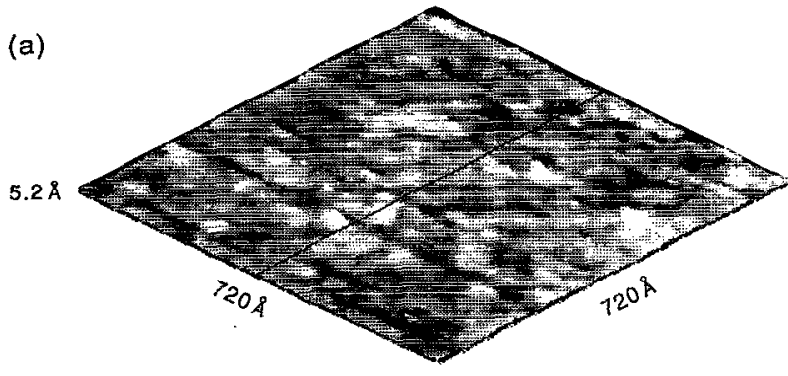

(b)
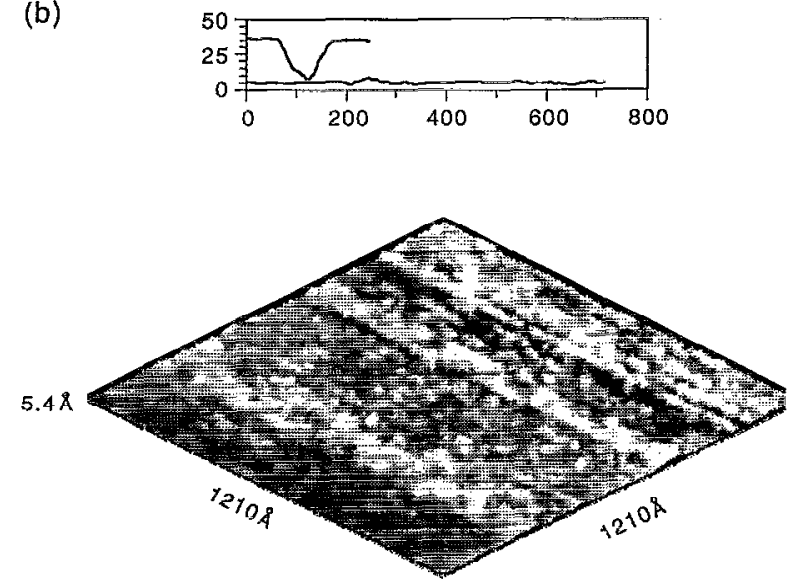

FIG. 4. Two surface regions of a 100 - $\AA$-thick $a$-Si:H film deposited on a GaAs substrate. The images are computer smoothed between $7 \AA$ spaced data points. These and all images are "deplaned," and unless noted otherwise the vertical scales are all shown three times magnified. A cross section along the line in (a) as well as the inverted image of a small hill in a nearby scan is shown in (b).

sionally observed on this surface. It is not clear if these were microparticulates deposited during film growth or during sample transfer, or simply growth structures. In either event, they provided a useful diagnostic of probe resolution; from their shape we estimate an upper limit on the probe tip radius. In Fig. 4(b) a contour through one such nearly cylindrical hill is shown inverted; the actual probe resolution, including the vacuum gap, must be sharper than this. This is compared to a line through the tallest and deepest features in the part (a) image, which was obtained in the next scan. The inverted hill is narrower than any features in this line, and we conclude that the topographies in parts (a) and (c) correctly represent most aspects of the film surface. However, the hill has a rounded tip on a $10 \AA$ scale, and surface pits of this size or smaller probably would have escaped detection.

Overall, the measured $100 \AA$ film surface topographies appear very flat, typically having two atomic layers $(5 \AA)$ of height variations in the form of $30-100 \AA$ wide lumps. There are no indications that islands have begun to form during this early stage of growth. $100 \AA$ films were also deposited on $n$-type Si (111) surfaces, prepared by Shiraki etching and thermal oxide removal in vacuum. Surface topographies on these films were generally consistent with Fig. 4, but less conclusive since some regions of the initial crystal surfaces were not atomically flat and clean. Since this initial perfect atomic flatness was not deemed neces- 


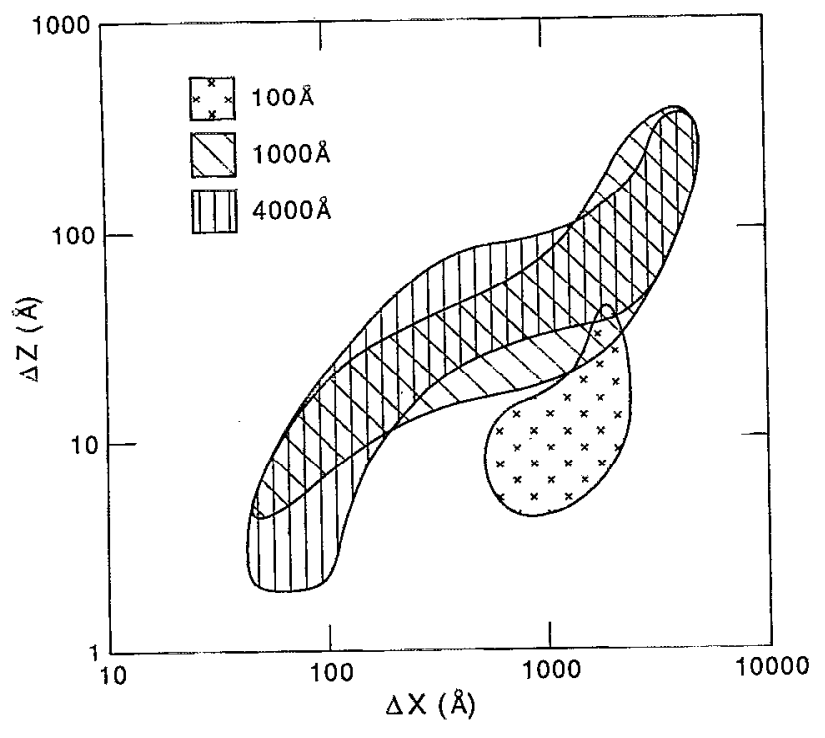

FIG. 5. The full height rangc $\Delta z$ (after deplaning) of various scanned surface regions, vs the size $\Delta x=\Delta y$ of the scanned region, for 100-, 1000-, and $4000-\AA$-thick films.

sary for thicker films, these films were deposited on crystal silicon to more closely mimic the standard film growth geometry used in photovoltaics.

\section{B. 1000- $\AA$ - and 4000- $\AA$-thick films}

Surface topographies on $1000 \AA$ films deposited on p-type, $20 \Omega \mathrm{cm}$, (100) Si and 4000- $\AA$-thick film deposited on $n$ type, $\sim 0.01 \Omega \mathrm{cm}, \mathrm{Si}$ (111) substrates will be shown here. These generally have a much rougher character and larger height range than the $100-\AA$-thick films shown above, and the topography of the $1000 \AA$ film is generally similar to that of the $4000 \AA$ film, Thus, much larger surface-height inhomogeneities appear to have developed between 100 and $1000 \AA$ of film growth, but the surface then reached a relatively stable form during further growth. We have observed many kinds of surface topographies and features; and we will discuss and provide a variety of examples of these. The total height range, $\Delta Z$, observed on each scan depends on the lateral scan size $\Delta X$ and varied considerably between different regions. In Fig. 5 we show contours that include the full range of $\Delta Z$ and $\Delta X$ values observed on $100-, 1000-$, and 4000 - $\AA$-thick films.

"Hilly" areas, consisting of apparently randomly placed, rounded hills covering the entire scanned region are found most often. Three examples are given in Fig. 6. The individual hills have rather low surface slopes, generally $<0.2$ from horizontal, and their lateral sizes vary from 50 to $2000 \AA$. The valleys between hills are generally broad and flat. As can be seen in Fig. 6, the typical size of these hills can vary greatly between different scanned regions, even on the same film; hills of successively larger size are shown in parts (a)-(c). Note that each edge of each figure provides a de facto scan line through the figure, but with slopes exaggerated three times since the vertical scales have been three times expanded. Based on detecting occasional
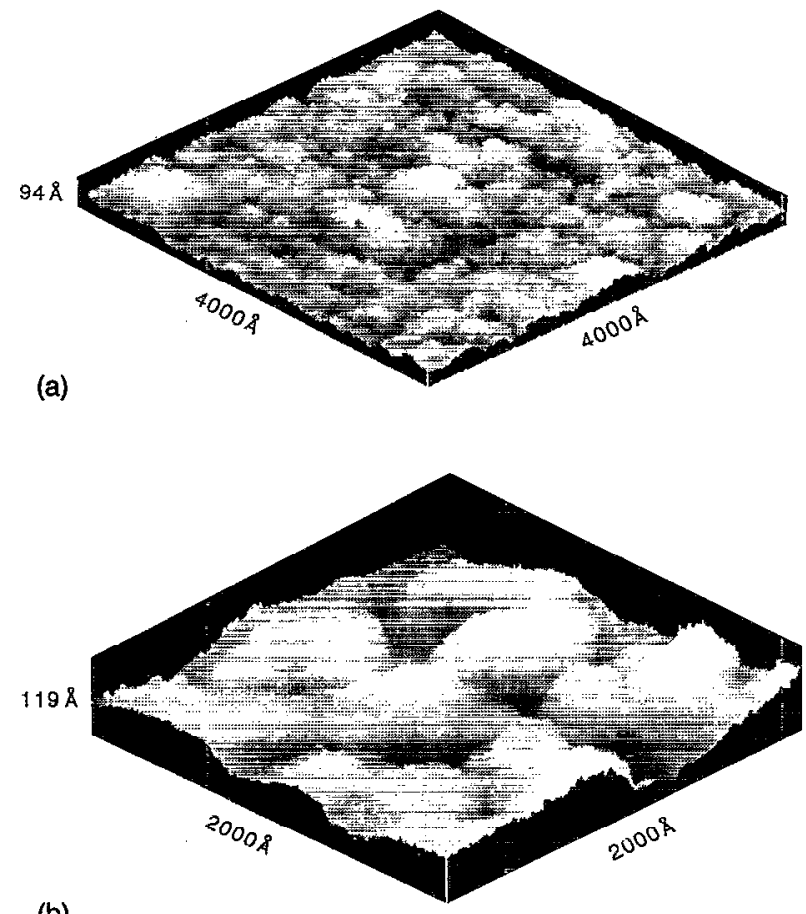

(b)

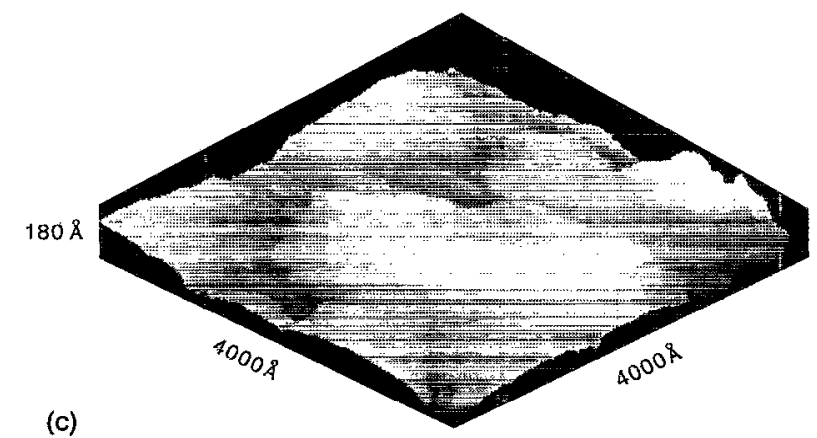

FIG. 6. Three examples of hilly surface regions, with varying hill size. (a) and (c) are from a $4000-\hat{A}$-thick films, and (b) is from a 1000-Â-thick film. Data points were taken at $10 \AA$ intervals.

hills that are steeper and smaller than any features in Fig. 6 , in other regions scanned before or after these areas (one of these is shown in Fig. 7), we believe that the topographies in Fig. 6 closely represent the large-scale surface shapes, without smearing by the probe resolution. Since height data were recorded at $10 \AA$ steps in these scans, and the small hills such as those in Fig. 7 have $10-20 \AA$ wide rounded tops, "large scale" here means $>20 \AA$.

A smail, steep hill is shown in lines $a-b$ and $c-d$ of Fig. 7 , which is a higher resolution scan $(2 \AA$ between data points) of a region nearby that in Fig. 6(b). The region in Fig. 7 may also be characterized as "hilly" on an even finer scale than in Fig. 6(a), but these smaller hills are not just a smaller version of the hills in Fig. 6(a). The latter are relatively smooth with low slopes and broad valleys between, whereas the small hills in Fig. 7 have steep sides and often narrow valleys. In fact, the data in Fig. 7 are undoubtedly smcarcd by probe resolution and the actual sur- 

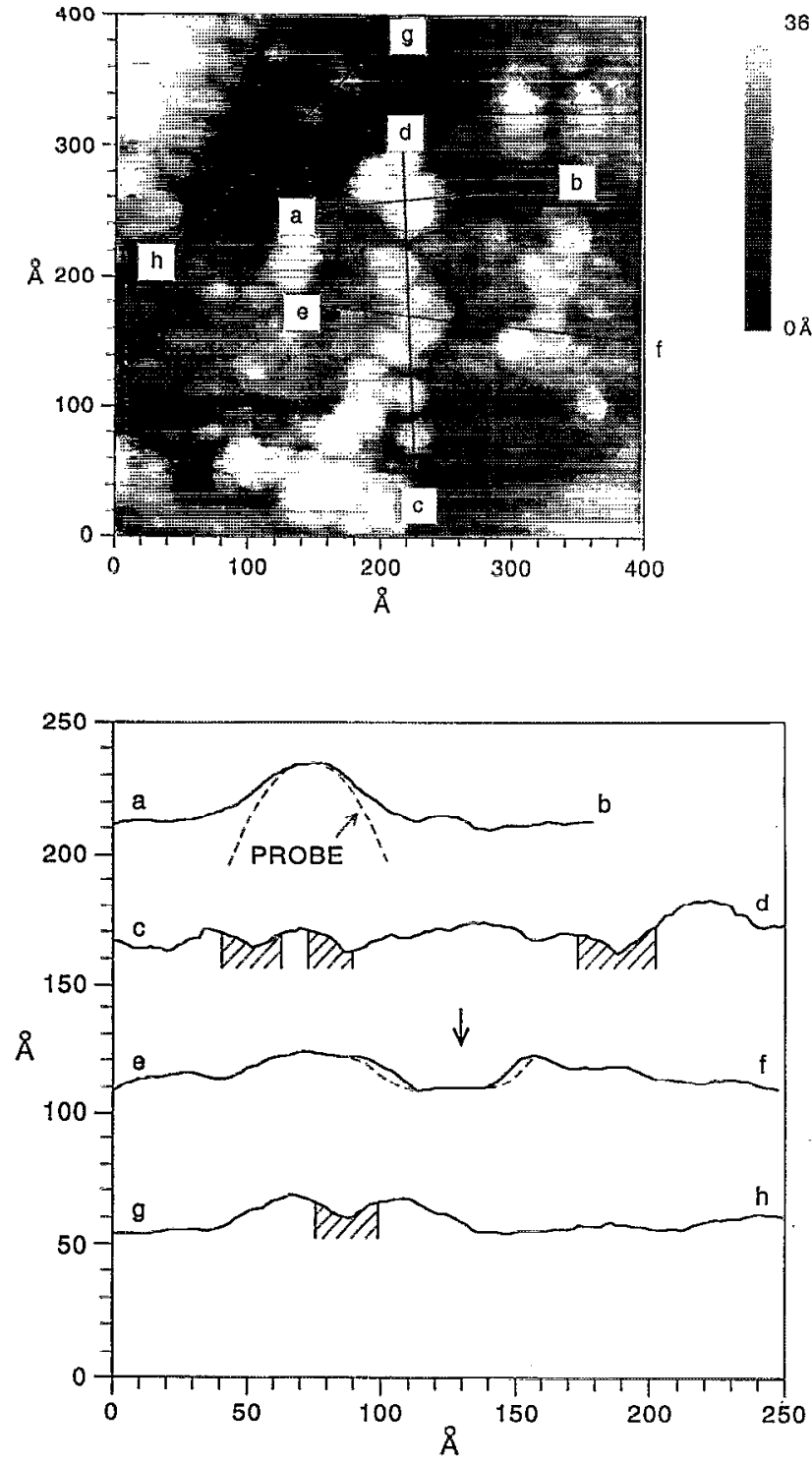

FIG. 7. A region of small, steep hills from a 1000 - $\AA$-thick film. Data points are at $2 \AA$ intervals. Part (b) shows cross-section lines through locations shown in (a). The probable probe shape is indicated under line $\mathrm{a}-\mathrm{b}$. Shaded regions indicate where the actual $a-\mathrm{Si}: \mathrm{H}$ surface could be below the scan line, due to the probe effect shown in line B of Fig. 3(a). The arrow in line e-f identifies a region that is similar to line A in Fig. 3 (a). The dashed lines on either side of the arrow indicate the probable a-Si:H surface.

face bumps are sharper than they appear. We will return to this figure below, in the context of incipient voids.

In complete contrast to the hilly areas described above, some surface regions are extremely smooth. Several examples are given in Figs. 8 and 9. The steepest slope across large regions of the surface in Fig. 8 is only 0.04 , and local regions of the surface appear to be extremely smooth. This overall smoothness is partly obscured by a probe-tip instability that caused 5-10 ̊ height fluctuations, but as these occur at random positions and generally form single scan line streaks, they can be readily distinguished from the actual surface. In fact, this instability is so clear in this figure precisely because the actual surface is exceedingly

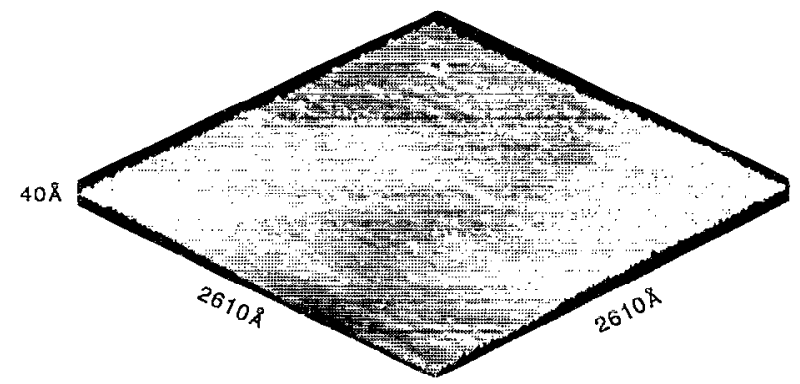

FIG. 8. An example of a smooth region of a 4000-Å-thick film. Data were recorded at $15 \AA$ intervals.

smooth, generally with less than $5 \AA$ height variations across 500 -Å-wide regions.

Figure 9 shows another flat region, as well as an exceptional view of the inhomogeneous character of the film surface. The left front quadrant of the $1000 \AA$ square region in Fig. 9(a) is hilly, with close-packed hills of $-.40 \AA$ height and $100 \AA$ width, having side slopes of $\sim 0.2$. The remaining three quadrants are flat within $5 \AA$, and as seen in Fig. 9(b) a $100 \AA$ square region at the front corner is flat within $2 \AA$. This degree of flatness on a 4000 - $\AA$-thick film is very impressive. It is important to note that because steep hills were observed in the same [part (a)] scan that showed the flat region, the flat portions of the image cannot be an artifact created by low probe resolution.

\section{Voids}

As noted in the introduction, many voids are believed to occur in $a$-Si:H films and to be important contributors to the electronic properties. ${ }^{10,11}$ In order to discern such voids in the act of forming at the surface, we have searched our data for relevant features. As discussed in Sec. III, all such

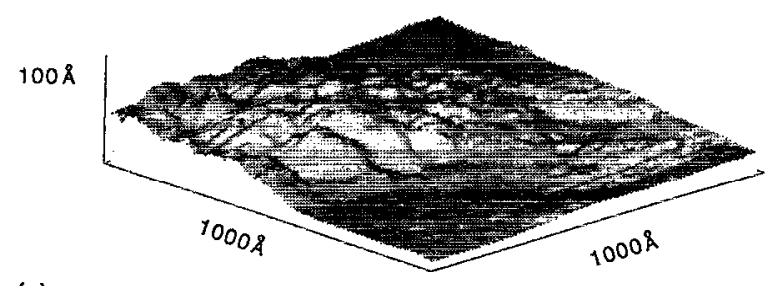

(a)

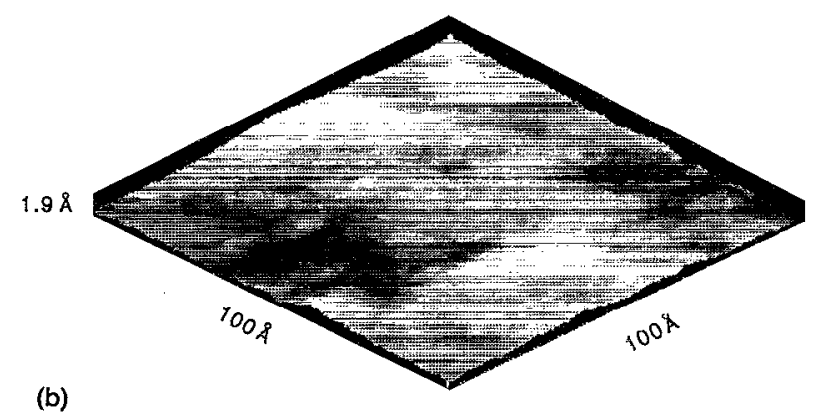

FIG. 9. A surface region of a $4000-\AA$-thick film, scanned at $10 \AA /$ point (a). In (b) is a $1 \AA$ /point scan of the $100 \AA$ square rcgion in the front corner of (a). 
low regions will be observed with a STM as narrower and often shallower than the actual shape, due to probe resolution. As shown in Fig. 3(a), the contact transform, at $\sim 5 \AA$ gap, of the inverted probe tip with a pit of comparable or smaller width will produce a cusp-shaped scan pit with a sharp point at the bottom. We have so far found two forms of such incipient voids; one form is a long canyon and the other is at low points between hills. An example of the former is given in Fig. 10, while Fig. 7 provides examples of the latter. Figure 10(a) shows a canyon, with observed depth of $\sim 20 \AA$. The cross-section line in Fig. 10(b) shows the cusp-like shape expected when the probetip diameter exceeds the canyon width; the canyon bottom is not reached in this case.

Figure 7(b) shows cross-section contours through a variety of the small hills in Fig. 7(a). These contours contain many cusp-shaped pits at the contacts between hills. As for the valleys in Fig. 10(b), the sides of these cusps are steeply sloped $\left(>45^{\circ}\right)$, further supporting the suggestion that these are incipient voids.

\section{DISCUSSION}

Intrinsic $a$-Si:H films, deposited by silane glow discharge, are known to be amorphous and comprised mainly of four fold-coordinated $\mathrm{Si}$ atoms with bond lengths and angles very close to crystalline values. However, the density is typically $5 \%$ below the crystalline density and typically contains $\sim 10 \%$ hydrogen atoms. The simplest way to reconcile these apparent contradictions is by postulating many small voids in the material, with $\mathrm{H}$ atoms bonded to the $\mathrm{Si}$ at these void surfaces. NMR and SAXS studies cited in Sec. I suggest that we actually are dealing with such an inhomogeneous material. The character of these voids may be crucial to the electronic properties and photovoltaic stability of these films, and finding the causes of void formation during film growth is a major concern. Although our data show incipient voids on the surface of this material, there is no attempt to measure a void density from these data. The nature of our data does not allow for estimates of the volume of our incipient voids nor whether they might be filled by further film growth. Finally, it should be noted that our recorded data are biased by interest in certain features and are therefore not valid for a statistical sampling. Here we report the first observations of the atomicscale topography over large areas of this growing surface of a device-quality film. (Prior STM studies concentrated on $\mathrm{H}$ atom bonding at the surface, based on tunneling barrier height, and in both cases heavily $p$-doped films were studied. In Ref. 17 the surface was also $\mathrm{Ar}^{+}$bombarded before tunneling.)

It is well established that $a-\mathrm{Si}: \mathrm{H}$ film growth from silane glow discharge results predominantly from $\mathrm{SiH}_{3}$ radical reactions with the film surface. ${ }^{3,4,28}$ These radicals diffuse through $\mathrm{SiH}_{4}$ gas to arrive at the surface in a cosine distribution, so they strike protruding regions of the surface more frequently than low regions. If they reacted and remained at the point of arrival, this microscopic shadowing would inevitably lead to a highly porous, filamentary and useless film. ${ }^{29}$ Since this is not observed, then either radical surface diffusion before incorporation occurs or energetic surface ion bombardment compacts the top layers of the growing film. Although such bombardment occurs for some deposition conditions, the highest (electronic) quality films are often grown under low-power glowdischarge conditions that yield minimal energetic bombardment. Thus, it is clear that considerable $\mathrm{SiH}_{3}$ surface diffusion must occur, and moreover that this diffusion must be accompanied by some degree of preferential incorporation in valleys as opposed to hilltops. On the other hand there appear to be many small voids in the final film, so this diffusion and preferential incorporation apparently fails to fill in many low regions before they are covered over. Understanding and ultimately controlling this void growth is a primary concern.

All models of the film growth have so far been homogeneous reaction-chemistry models, yet film inhomogeneities appear to be common and crucial. Other important, unsuspected factors must cause this inhomogeneous growth. To search for clues, the present study has surveyed many areas of a number of films and film thicknesses. We have looked for differences as well as patterns and found both. The most common surface shape found on fully developed films ( $>1000 \AA$ thickness) was continuous rounded hills with height/width $<0.2$, gradually sloping sides and relatively flat connecting valleys. The surfaces of the larger hills $(\sim 100 \AA$ height, $\sim 1000 \AA$ width $)$ were often covered with finer hills, but again with low height/ width and slopes. No incipient voids are indicated in such regions, although those of $<20 \AA$ size would probably have been missed due to our probe resolution.

Several major variations on the above "gentle rolling hill" regions were found. In some regions steep sided, small ( $\sim 50$-A-wide) hills were observed, with narrow, steep pits suggesting incipient voids at their intersections (Fig. 7). Steep-sided canyons through other regions clearly indicate long, connected incipient voids (Fig. 10). These might even have been the edge of an internal void surface. One unusually large hill $(\sim 300 \AA$ high, $\sim 2000 \AA$ wide, not shown here) was observed on an otherwise relatively flat area, suggesting a $300-\AA$-tall microparticulate transformed with the probe tip. This and many smaller but relatively steep-sided hills (e.g., Fig. 7) might be attributed to silicon microparticulates deposited from the discharge, if we assume that these can conduct the STM current or be covered by a conducting film. On the other hand, any region that can sustain more rapid than average growth through a number of layers will produce a similar image with our present STM resolution.

The most surprising deviations from the overall hilly character of the fully developed $(\geqslant 1000 \AA$ ) surface were the very smooth regions. These areas were observed to have at most a few atomic layers of height variation across hundreds of $\AA$ in lateral dimensions (e.g., Figs. 8 and 9). Generally, this was a smooth surface on a large, gently sloping hill (e.g., Fig. 8), rather than a nearly constant film thickness. These areas appear extremely homogeneous and free of voids (of $>10 \AA$ size), and if the entire film had this character it might have superior properties. These 
(a)

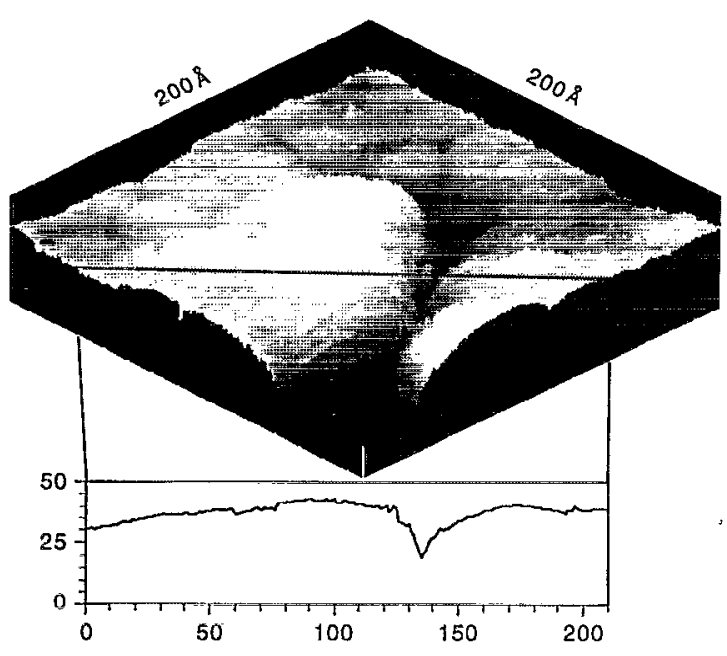

FIG. 10. Shaded view and cross section of a surface region of a $4000-\AA \AA-$ thick film, showing a steep, narrow canyon.

areas are not crystalline, as $a$-Si:H does not grow microcrystals under our deposition conditions. ${ }^{30}$ Very effective $\mathrm{SiH}_{3}$ diffusion and valley filling is demanded to explain the existence of such regions. But if this is true, then other factors are required to explain the failure to smooth out other areas. This dichotomy of smooth versus steep, hilly areas on the same surface is uniquely demonstrated by Fig. 9, where the border between such a smooth and a hilly region was captured.

We have so far observed with moderate probe resolution relatively few $a$-Si:H films, all deposited under a single set of discharge conditions. Although limited, these data nonetheless support the model of $\mathrm{SiH}_{3}$ diffusion and preferred valley incorporation, at least in many areas of the surface. However, these observations also show that the film surface is highly inhomogeneous, suggesting that other crucial factors are playing a role in the growth of film voids and inhomogeneities. It is tempting to conjecture on possible causes, to see if these might suggest ways to improve the photovoltaic quality and stability of intrinsic $a-\mathrm{Si}: \mathrm{H}$. We offer a few ideas here.

(1) In order to produce the ( $>20$ - $\AA$-wide) structural inhomogeneities observed here, an irregularity at one surface atomic site or in one surface region must influence the growth of many overlying atomic layers; it cannot be buried and restored to normal by the next Si layer. This might be achieved by a surface impurity that has enhanced reactivity for $\mathrm{SiH}_{3}$, but only if the incorporation reaction frequently transfers the impurity up to the new surface. This might be possible for surface-bonded $\mathrm{OH}$ or $\mathrm{NH}_{2}$ radicals, for example. The average impurity concentration in intrinsic $a$-Si:H films is $\sim 10^{18} / \mathrm{cm}^{3}$, far more than needed to induce reported void densities, but most studies of intrinsic $a$-Si:H electronic properties suggest minimal correlation with impurity concentrations (for reasonably low values).

(2) Crystal-growth rates often vary considerably in different directions and at step edges, and such orientation effects might also occur for $\mathrm{SiH}_{3}$ reactions with $\mathrm{H}$-covered film. The short-range order in $a$-Si:H may be sufficient to maintain effective orientations across nm scale distances, and this could lead to unequal growth rates over a similar thicknesses range. This could combine with incomplete diffusional compensation for shadowing to yield many small voids.

(3) One of the most obvious potential causes of small voids is a nonreactive surface site. $\left(\mathrm{A} \mathrm{CH}_{3}\right.$ attached to a $\mathrm{Si}$ surface atom is a well-known example in $a$-Si:C:H alloy films; the $\mathrm{CH}_{3}$ is usually buried intact. ${ }^{31}$ ) Shadowing will tend to expand and propagate this void upward through successive $\mathrm{Si}$ layers, while $\mathrm{SiH}_{3}$ diffusion counters this. Only in the unlikely event that reactivity at adjacent sites is also lowered should this lead to more than a very small void, essentially enclosing the nonreactive site.

(4) Microparticulates, which are presumed chemically similar but electronically different than the film, can form in the discharge and bond to the surface. ${ }^{32,33} \mathrm{It}$ is unlikely that $\mathrm{SiH}_{3}$ diffusion is sufficient to fill in the gaps below and adjacent to such particulates, so voids of comparable size would probably result. However, these particulates become negatively charged once they reach perhaps $10 \AA ̊$ size, and gravity, gas flow, and their charge tend to keep them away from the substrate. Also, particulates have not been detected in low power, high flow discharges such as used here.

None of the above conjectures offers feasible explanations of the canyon-like incipient voids we detected (Fig. 10). These suggest an entire void surface reaching down into the film, or at least a cord-like void. This appears to require the coalescence of many void activators (impurities?), or a nonreactive boundary between growing regions. Although this looks somewhat like a finer-scale form of "columnar" films, ${ }^{8,9}$ we do not think these are directly related. Those columnar films were grown in very different discharges under "physical deposition" conditions, where shadowing effects dominate and minimal $\mathrm{SiH}_{3}$ deposition occurred. Perhaps in the present case impurities tend to coalesce into such regions, preserving diminished growth rates. $\mathrm{SiH}_{3}$ incorporation on surfaces facing the plasma might be enhanced relative to inside deep canyons, causing small voids to grow laterally as well as upward with additional layers.

\section{CONCLUSIONS}

This STM-based investigation of $a$-Si:H surface topographies has discerned a variety of film characteristics that appear significant to its electronic properties and stability. Perhaps the most surprising and important observation is significant film inhomogeneity found by comparing different surface regions; the films appeared to be a multiphase material. Void regions appeared to be clustered, with some appearing at the surface as a line, as might be expected for a grain boundary. Other surface areas were very flat, and the lateral size of surface hills varied between regions. We could only conjecture from this initial study using one set of deposition conditions what might be responsible for these film variations. However, the mere existence of extremely smooth and almost atomically flat surface regions 
supports the $\mathrm{SiH}_{3}$ diffusion and valley-filling model, and also offers a tantalizing hope that it might be possible to produce more homogeneous and perhaps better photovoltaic material.

A significant difference was observed between thin films of $100 \AA$ and thicker films of 1000 and $4000 \AA$. The thin films show no sign of islanding, particulates, or void structures, and appeared much like the smoothest regions of the thicker films. Another useful result of this study is a demonstration of the ability to vacuum tunnel into intrinsic $a$-Si:H without damaging the material. Occasional atomic transfers between sample and probe, and tunneling gap fluctuations clearly occurred, but reproducible topographies were generally obtained.

\section{ACKNOWLEDGMENTS}

The authors would like to thank A. Laracuente for help in conducting these experiments. This work has been supported by the National Renewable Energy Laboratory under Contract No. DD-1-11001-1. Additionally, D. Tanenbaum acknowledges the support of a Department of Energy University-Laboratory Graduate Fellowship.

${ }^{1}$ H. M. Branz, R. S. Crandall, and M. Silver, AIP Conf. Proc. 234, 29 (1991).

${ }^{2}$ M. J. Kushner, J. Appl. Phys. 63, 2532 (1988); 62, 4763 (1987).

${ }^{3}$ R. Robertson and A. Gallagher, J. Appl. Phys. 59, 3402 (1986).

${ }^{4}$ A. Gallagher, J. Doyle, and D. Doughty, Mater. Res. Soc. Symp. Proc. 149, 23 (1989).

${ }^{5}$ D. A. Doughty, J. R. Doyle, G. H. Lin, and A. Gallagher, J. Appl. Phys. 67, 6220 (1990).

${ }^{6}$ J. L. Guizot, K. Nomoto, and A. Matsuda, Surf. Sci. 244, 22 (1991).

${ }^{7}$ J. Perrin and T. Broekhuizen, Appl. Phys. Lett. 50, 433 (1987).

${ }^{8}$ J. C. Knights and R. A. Lujan, Appl. Phys. Lett. 35, 244 (1979); J. Knights, J. Non-Cryst. Solids 35/36, 159 (1980).

${ }^{9}$ R. C. Ross, A. G. Johncock, and A. R. Chen, J. Non-Cryst. Solids 66, 81 (1984).

${ }^{10} \mathrm{~J}$. A. Reimer and M. A. Petrich, in Advances in Disordered Semiconductors, edited by H. Fritzsche (World Scientific, Singapore, 1989), Vol. 1, p. 3.
${ }^{11}$ A. H. Mahan, D. L. Williamson, B. P. Nelson, and R. S. Crandall, Phys. Rev. B 40, 12024 (1989).

${ }^{12}$ B. Drevillon, Thin Film Solids 130, 165 (1985).

${ }^{13}$ R. W. Collins and J. M. Canese, J. Appl. Phys. 61, 1869 (1987).

${ }^{14}$ Y. Toyoshima, K. Arai, A. Matsuda, and K. Tanaka, Appl. Phys. Lett. 56, 1540 (1990).

${ }^{15}$ G. H. Lin, J. R. Doyle, M. He, and A. Gallagher, J. Appl. Phys. 64, 188 (1988).

${ }^{16}$ L. L. Kazmerski, Ninth E. C. PV Solar Energy Conference, Freiburg, FRG, 25 September 1989. Also see 1989 SERI Annual Report under Contract No. DE-AC02-83 CH-100-93, NREL, 1617 Cole Blvd., Golden, CO 80401.

${ }^{17}$ R. Wiesendanger, L. Rosenthaler, H. R. Hidber, H. J. Güntherodt, A. W. McKinnon, and W. E. Spear, J. Appl. Phys. 63, 4515 (1988).

${ }^{18}$ J. Jahanmir, P. E. West, S. Hsieh, and T. N. Rhodin, J. Appl. Phys. 65, 2064 (1989).

${ }^{19}$ E. Hartmann, R. J. Behm, G. Krötz, G. Müller, and F. Koch, Appl. Phys. Lett. 59, 2136 (1991).

${ }^{20}$ G. J. Vandentop, P. A. P. Nascente, N. Kawasaki, D. F. Ogletree, G. A. Somorjai, and M. Salmeron, J. Vac. Sci. Technol. A 9, 2273 (1991).

${ }^{21}$ J. W. Lyding, S. Skala, J. S. Hubacek, R. Brockenbrough, and G. Gammie, Rev. Sci. Instrum. 59, 1897 (1988).

${ }^{22}$ A. Ishizaka and Y. Shiraki, J. Electrochem. Suc. 133, 666 (1986).

${ }^{23}$ R. W. Collins, C. J. Tuckerman, C.-Y. Huang, and H. Windischmann, J. Vac. Sci. Technol. A 3, 2077 (1985).

${ }^{24}$ J. A. Stroscio, R. M. Feenstra, and A. P. Fein, Phys. Rev. Lett. 57, 2579 (1986).

${ }^{25}$ M. B. Johnson and J.-M. Halbout, J. Vac. Sci. Technol. B 10, 508 (1992).

${ }^{26}$ D. Keller, Surf. Sci. 253, 353 (1991).

${ }^{27}$ D. A. Grigg, J. E. Griffith, G. P. Kochanski, M. J. Vasile, and P. E. Russell, Proc. SPIE 1673, 557 (1992).

${ }^{28} \mathrm{~K}$. Tachibana, A. Yuuki, and Y. Matsui, in Gaseous Electronics and its Applications, edited by R. W. Crompton, M. Hayashi, and D. E. Boyde (Kluwer, Dordrecht, 1991), p. 273.

${ }^{29}$ K. K. Gleason, K. S. Wang, M. K. Chen, and J. A. Reimer, J. Appl. Phys. 61, 2866 (1987).

${ }^{30}$ G. Ganguly, C. De, S. Ray, and A. K. Barua, J. Appl. Phys. 69, 3915 (1991), and references therein.

${ }^{31} \mathrm{~J}$. Sotiropoulos and G. Weiser, J. Non-Cryst. Solids 92, 95 (1987), and references therein.

${ }^{32}$ L. Boufendi, A. Plain, J. Ph. Blondeau, A. Bonchoule, C. Taure, and M. Toogood, Appl. Phys. Lett. 60, 169 (1992).

${ }^{33}$ A. Bouchoule, A. Plain, L. Boufendi, J. Ph. Blondeau, and C. Laure, J. Appl. Phys. 70, 1991 (1991). 